\title{
One-stage definitive repair of pulmonary atresia with intact ventricular septum and hypoplastic right ventricle
}

Takeshi Shinkawa, MD, Masaaki Yamagishi, MD, Keisuke Shuntoh, MD, Keitarou Koushi, MD, Takahiro Hisaoka, MD, and Hitoshi Yaku, MD, Kyoto, Japan

$\mathrm{T}$ he right ventricle (RV) overhaul technique is an effective method for enlarging the RV cavity on the way to biventricular repair for pulmonary atresia and intact ventricular septum with hypoplastic RV. We describe two successful cases of one-stage definitive repair with the RV overhaul technique in early infancy.

\section{Clinical Summaries}

The first case was that of a 1-month-old boy weighing $3.3 \mathrm{~kg}$. His preoperative right ventriculogram showed tripartite RV with enddiastolic volume $50 \%$ of the normal value, ${ }^{1}$ a tricuspid valve with annular diameter $67 \%$ of the normal value $(Z$ value -2.2$),{ }^{2}$ and minor sinusoidal communications. The second case was that of a 1-month-old girl weighing $3.1 \mathrm{~kg}$. Her preoperative right ventriculogram showed tripartite RV with end-diastolic volume $67 \%$ of the normal value, a tricuspid valve with annular diameter $101 \%$ of the normal value $(Z$ value +0.3$)$, and no sinusoidal communication (Figures 1, A, and 2, A). The pulmonary circulation was ductus dependent, and the intervention to perforate atretic pulmonary valve was unsuccessful in both cases.

In both cases cardiopulmonary bypass was established through a median sternotomy and the ductus was divided. Severe tricuspid valvular dysplasia was not detected in either case. In case 1, the pulmonary arterial incision was extended to the ventricular outflow portion because the pulmonary annular diameter was too small. In case 2 , pulmonary valvotomy was performed because the pulmonary annular diameter was of approximately normal size. The hypertrophied muscles in the outflow and the trabecular portions were resected as possible (RV overhaul). In case 1, the outflow tract incision was patched with a monocuspid pericardial patch. A purse-string suture was placed around the foramen ovale and extended to the outside of the heart. After weaning from bypass, the foramen ovale was closed after test snaring of the purse-string

From the Department of Pediatric Cardiovascular Surgery, Children's Research Hospital, Kyoto Prefectural University of Medicine, Kyoto, Japan.

Received for publication May 12, 2005; accepted for publication June 7, 2005.

Address for reprints: Takeshi Shinkawa, MD, Department of Pediatric Cardiovascular Surgery, Children's Research Hospital, Kyoto Prefectural University of Medicine, Kawaramachi, Hirokoji, Kamigyo-ku, Kyoto, 6028566 Japan (E-mail: newriver@koto.kpu-m.ac.jp).

J Thorac Cardiovasc Surg 2005; 130:1207-8

$0022-5223 / \$ 30.00$

Copyright $\odot 2005$ by The American Association for Thoracic Surgery doi:10.1016/j.jtcvs.2005.06.009
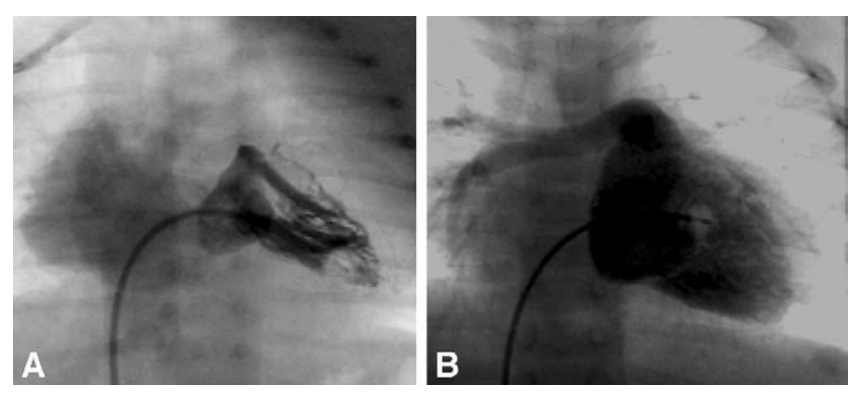

Figure 1. Preoperative (A) and postoperative (B) right ventriculograms from case 1 showing enlarged RV (126\% normal enddiastolic volume) and grown tricuspid valve (79.3\% normal annular size).
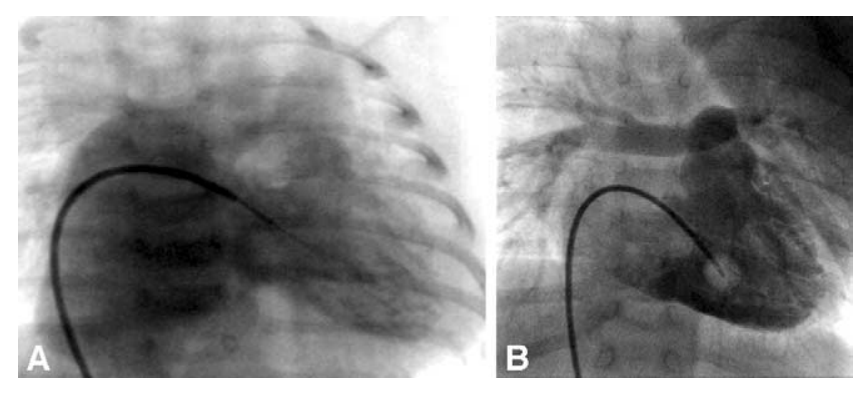

Figure 2. Preoperative (A) and postoperative (B) right ventriculograms from case 2 showing enlarged RV $(128 \%$ normal enddiastolic volume) and competent tricuspid valve $(80.0 \%$ normal annular size).

suture. Postoperative right ventriculograms showed competent tricuspid valves and adequately enlarged right ventricles (Figures 1, $B$, and $2, B$ ). The right atrial pressures were 11 and $7 \mathrm{~mm} \mathrm{Hg}$ in cases 1 and 2, respectively.

\section{Discussion}

Most patients with pulmonary atresia and intact ventricular septum with a tripartite RV and an absence of RV-dependent coronary circulation are initially treated with pulmonary valvotomy, irrespective of RV volume. ${ }^{3,4}$ However, it has been reported that the tricuspid valve diameter does not change after the initial valvotomy, ${ }^{4}$ and the size of the tricuspid valve would be a risk factor for not receiving biventricular repair. ${ }^{2}$ We have therefore formulated a new strategy, consisting of the RV overhaul tech- 
nique and concomitant RV outflow tract reconstruction in early infancy to achieve greater tricuspid valve and RV development for patients who cannot undergo successful catheter intervention. We believe that the postoperative tricuspid valve and RV can receive more venous return and have greater growth potential with this strategy than with simple valvotomy. If vital signs became unstable after test snaring of the interatrial communication, the communication should be left open. This strategy seems to be appropriate for patients with tripartite RV and tricuspid annulus larger than $60 \%$ of normal value without severe valvular dysplasia.

\section{References}

1. Rowlatt UF, Rimoldi HJ, Lev M. The quantitative anatomy of the normal child's heart. Pediatr Clin North Am. 1963;10:499-588.

2. Hanley FL, Sade RM, Blackstone EH, Kirklin JW, Freedom RM, Nanda NC. Outcomes in neonatal pulmonary atresia with intact ventricular septum. A multiinstitutional study. J Thorac Cardiovasc Surg. 1993; 103:406-27.

3. Pawade A, Capuani A, Penny DJ, Karl TR, Mee RB. Pulmonary atresia with intact ventricular septum: surgical management based on right ventricular infundibulum. J Card Surg. 1993;8:371-83.

4. Sano S, Ishino K, Kawada M, Fujisawa E, Kamada M, Ohtsuki S. Staged biventricular repair of pulmonary atresia or stenosis with intact ventricular septum. Ann Thorac Surg. 2000;70:1501-6.

\title{
The "clover technique" as a new approach for correction of postendocarditic severe tricuspid valve regurgitation
}

\author{
Georges Fayad, MD, ${ }^{a}$ Thomas Modine, MD, ${ }^{a}$ Pierre-Vladimir Ennezat, MD, ${ }^{b}$ Thierry Le Tourneau, MD, PhD, ${ }^{a}$ \\ Benoît Larrue, MD, ${ }^{a}$ Richard Azzaoui, MD, ${ }^{\text {a }}$ Olivier Nugue, MD, ${ }^{\mathrm{b}}$ Olivier Leroy, MD, ${ }^{\mathrm{c}}$ Christophe Decoene, MD, \\ Philippe Asseman, MD, and Henri Warembourg, MD, ${ }^{\text {a }}$ Lille and Tourcoing, France
}

\section{$\mathrm{V}$}

arious surgical treatments applied to tricuspid endocarditis provide immediate satisfactory results but seem to offer poor midterm results. We here report the use of the clover technique ${ }^{1}$ as a good alternative.

\section{Patient and Methods}

A 31-year-old male patient with a history of intravenous heroin addiction and active hepatitis $\mathrm{C}$ was admitted in a septic status with consciousness disorder and acute respiratory insufficiency.

A full-body scan revealed several pulmonary and splenic abscesses. Transesophageal echocardiographic findings showed massive vegetation on the mitral valve posterior commissure (Figure 1, $A$ ) with a grade II mitral valve incompetence. There were several vegetations on the tricuspid valve with a grade II leak. Hemocultures had revealed a meti-R Staphylococcus aureus. Intravenous antibiotic treatment with cefotaxime, fosfomycin, rifampicin, and fluconazole was initiated. A positive pulmonary and biologic outcome was observed rapidly. On the contrary, echocardiographic features worsened, with increasing mitral vegetation and grade IV mitral leak together with a grade IV tricuspid leak caused by total

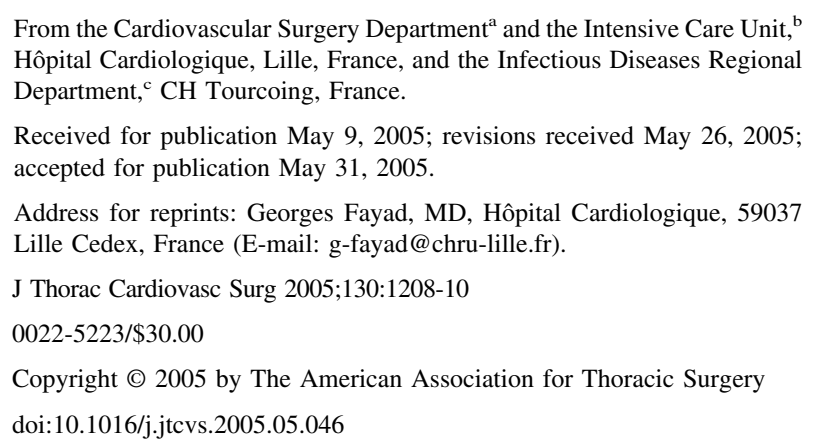

dehiscence of the anterior cusp. With respect to this development, we decided to operate.

The intervention was performed under extracorporeal circulation at $33^{\circ} \mathrm{C}$. The mitral valve was approached through a left atriotomy with resection of the posterior commissure and A3 and P3 sections and restoration of this area with a pericardial patch sutured to the posterior papillary muscle with a 4-0 polytetrafluoroethylene thread (Gore-Tex; W. L. Gore \& Associates, Inc, Flagstaff, Ariz), producing satisfactory results (Figure 1, B).

Analysis of the tricuspid valve confirmed dehiscence of the anterior cups and the absence of vegetations. We performed an annuloplasty according to the clover technique ${ }^{1}$ reinforced with an MC3 Edwards ring no. 36 (Edwards Lifesciences, Irvine, Calif; Figure 2).

Perioperative transesophageal echocardiography confirmed satisfactory results.

The postoperative course was uneventful, with continuation of the intravenous antibiotic treatment for 3 weeks, followed by oral delivery of rifampicin and pristinamycin (Pyostacine) for a further 3 weeks. The valve culture was sterile.

At the 6-month follow-up, the patient was in good general condition and in New York Heart Association functional class I, and no mitral or tricuspid leak was observed at echocardiography. Mean gradients were 6 and $4 \mathrm{~mm} \mathrm{Hg}$ for the tricuspid and mitral valves, respectively. An exercise test was carried out, confirming a good cardiovascular tolerance to effort.

\section{Discussion}

Tricuspid valve endocarditis benefits from antibiotic treatment with generally satisfactory results. ${ }^{2}$ Medical treatment is preferred because of the poor midterm results obtained with various surgical options. In this case report the surgical approach was considered 